\title{
Analysis of RTN signals in Resistive-Switching RAM device and its correlation with device operations
}

\author{
W. Zhang, Z. Chai, J. Ma, J. F. Zhang, Z. Ji \\ Department of Electronics and Electrical Engineering, Liverpool John Moores University, Liverpool L3 3AF, UK \\ Corresponding author, email: w.zhang@ljmu.ac.uk
}

\begin{abstract}
Filament rupture/restoration induced by movement of defects, e.g. oxygen ions/vacancies, is considered as the switching mechanism in $\mathrm{HfO}_{2}$ RRAM. However, details of filament alteration during switching are still speculative, due to the limitations of existing experiment-based probing techniques, impeding its understanding. In this work, for the first time, an RTN-based defect tracking technique is developed for RRAM devices, which can monitor the movements of defects and statistically provide their spatial and energy profiles. The critical filament region is experimentally identified and its alteration is observed and correlated with switching operations under various operation conditions. This provides a useful tool for further development of RRAM technology.
\end{abstract}

\section{Introduction}

Resistive switching random access memories (RRAM) are considered as a disruptive technology [1-3]. This is enabled by its sub-ns fast operation, sub-pJ low power consumption, $10^{6} \sim 10^{9}$-cycle endurance, sub-10 nm scalability and 3-D stack-ability [2-3]. It also has great potential to revolutionize the FPGA reconfigurable logic due to its nanoscale simple Metal-Insulator-Metal memory structure [4] and neuromorphic artificial intelligent computing due to its natural neurological performance similar to biologic synapses [5]. Valence Change Memory (VCM) is an important category of such devices, in which the resistive switching is controlled by the drift of anions, typically oxygen and/or its vacancy $(\mathrm{Vo})$ in the insulator, i.e., an thin oxide dielectric layer, inducing local valence change in the atomic lattice [6]. In filament-based VCM devices, information is stored in the form of variable resistance induced by repeatable rupture and restoration of a conductive filament (CF) of nanometer scale. For example, bipolar switching behavior has been reported in many binary as well as ternary oxides such as $\mathrm{HfO}_{2}$, $\mathrm{Ta}_{2} \mathrm{O}_{5}, \mathrm{Al}_{2} \mathrm{O}_{3}, \mathrm{TiO}_{2}, \mathrm{SrTiO}_{3}$, etc., in which it is believed that $\mathrm{CF}$ can be formed throughout the oxide layer under a positive voltage, lowering the resistance of the device into a low resistive state (LRS), and can be ruptured by applying a negative voltage, returning to the high- resistance-state (HRS). It is therefore of critical importance to gain the insight details of the $\mathrm{CF}$ modification process.

Whilst promising results have been achieved by tremendous globe-wide efforts in the past decade, details of the underlying microscopic picture are still largely missing, mostly because the CF modification occurs in a localized region of nano-meter scale surrounded by conducting electrodes and insulating dielectric, hindering its characterization. High-resolution physical analysis techniques have been used to provide information of the $\mathrm{CF}$, such as $3 \mathrm{D}$ tomography with conductive atomic force microscopy [7], in-situ transmission electron microscopy [8] and scanning tunnelling microscopy [9], which, however, are either destructive or require specially prepared test samples, and the tests are time consuming and statistically unfriendly. Experimental details of CF modification at Vo level are still missing. For example, it is not clear how Vo migrates under various operating conditions, both individually and statistically, and hence it is not clear how it contributes to the CF alteration between HRS and LRS. Direct experimental evidence of $\mathrm{CF}$ modification at Vo level should provide insight into the critical filament rupture/restore region and enhance our level of understanding on the switching mechanism. In this work, a random telegraph noise (RTN) based defect tracking technique (RTD) has been developed that can detect individual Vo movement and provide statistical information of filament alteration in both spatial and energy terms during normal operations in nanoscale RRAM devices, for the first time.

\section{Devices and Experiments}

The RRAM device under investigation is a TiN/Hf/HfO /TiN memory cell patterned into a crossbar shape to achieve the smallest area whilst minimizing the impact of parasitic elements [2]. The $\mathrm{Hf} / \mathrm{HfO}_{2}$ crossbar memory element are formed at the intersection between the bottom and top TiN electrodes, achieving $40 \mathrm{~nm} \times 40$ $\mathrm{nm}$ cell size. The 5-nm-thickness $\mathrm{HfO}_{2}$ dielectric, deposited on a planarized TiN surface by atomic layer deposition (ALD) using $\mathrm{HfCl}_{4}$ and $\mathrm{H}_{2} \mathrm{O}$ as precursors, is mainly amorphous. On top of the hafnia film, the 
10-nm-thickness Hf metal-cap was deposited by physical vapor deposition (PVD) acting as an oxygen reservoir during the device operations, which allows increasing the concentration of mobile defects (Vo) in $\mathrm{HfO}_{2}$, obtaining O-deficient, sub-stoichiometric $\mathrm{HfO}_{2-\mathrm{x}}$, thus enabling the ionic and electronic behavior required for resistive switching. After an initial electroforming process to form the $\mathrm{HfO}_{2-\mathrm{x}}$ filament, the typical current-voltage characteristic exhibits a stable bipolar resistive-switching behavior, i.e., SET to LRS at a positive bias, and RESET to HRS at a negative bias. The maximum current through the device is limited during the SET at a compliance current level between $50 \mu \mathrm{A}$ and $150 \mu \mathrm{A}$ to avoid device breakdown.

\section{Results and discussions}

\subsection{RTN signals in RRAM}

In order to investigate the mobile defects (Vo) that result in the $\mathrm{CF}$ without destructing the device, we use the random telegraph noise signals (RTN) as the electrical measurement tool $[10,11]$. The origin of RTN is attributed to the random electron trapping and detrapping process in a defect located in the oxide, leading to two discrete, high and low, current levels, as shown in Fig.1a. The bias dependence of mean $\tau_{\mathrm{c}}$ and $\tau_{\mathrm{e}}$ has been one of the main experimental evidences used in MOSFETs allowing not only the extraction of defect location and energy level but also the investigation of the mechanisms of electron transferring between the electrode and a single oxide trap (Fig.1b and eq.1\&2).

Fewer efforts have been made to investigate the RTN in RRAM because complex signals are often observed to which the above equations are not applicable. For example, defect movement (Vo) and electron trapping/detrapping can co-exist [3], both of which can result in current fluctuation and lead to complex RTN; Moreover, Equations (1)-(2) are only applicable to the cases that an electron tunnels from an electrode to an oxide defect, and then back to the same electrode, in which $\tau_{\mathrm{c}}$ and $\tau_{\mathrm{e}}$ should have the opposite polarity of bias dependence. However, due to the nanoscale oxide thickness in RRAM devices, RTN can also results from electron tunneling through the defect to the opposite electrode or to other defects, in which cases $\tau_{\mathrm{c}}$ and $\tau_{\mathrm{e}}$ show the same polarity of bias dependence (Fig.2a). There also might be a large quantity of defects near each electrode, which leads to metallic-like local conduction (Fig.2b). To avoid these complications, in this work, we only consider RTN that clearly follows the classical theory eq. $1 \& 2$, as shown in Fig. $1 \mathrm{a} \&$ b. that $\tau_{\mathrm{c}}$ and $\tau_{\mathrm{e}}$ have the opposite polarity of bias dependence. This ensures
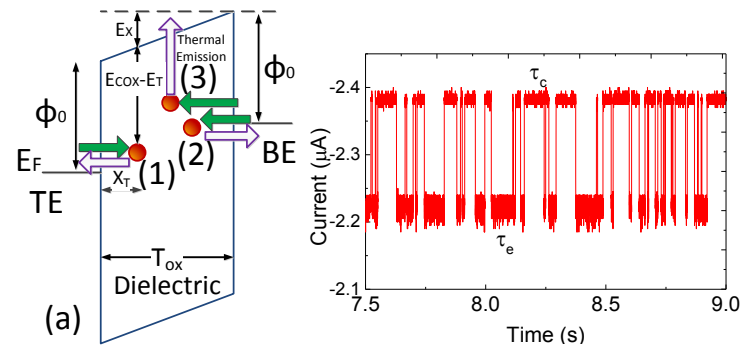

$\frac{X_{T}}{T_{O X}}=\frac{K_{B} T}{q} \frac{\partial}{\partial V}\left[\ln \left(\frac{\tau_{c}}{\tau_{e}}\right)\right]$

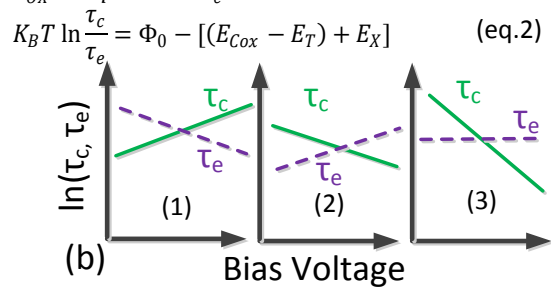

Fig.1 (a) Energy band diagram illustrating various RTNs examined in this work caused by electron tapping and detrapping, and a typical RTN signal. (b) Defect's spatial and energy location $\left(\mathrm{X}_{\mathrm{T}}, \mathrm{E}_{\mathrm{T}}\right)$ can be extracted from the capture and emission time constant dependence on the bias for these RTNs by using eq. $1 \& 2$ [11,12].
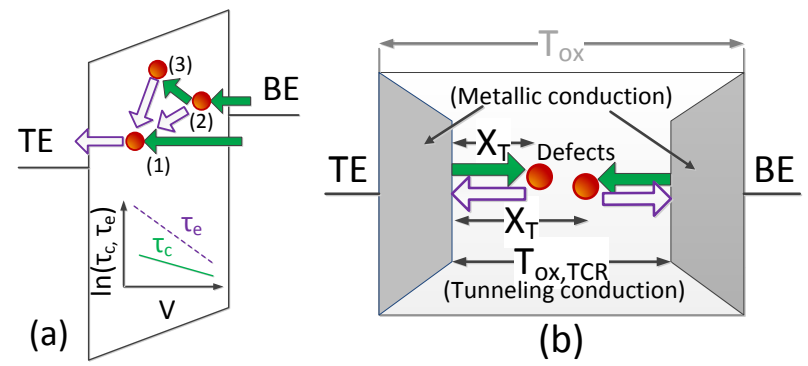

(b)

Fig.2 Eq.1\&2 are not applicable for (a) RTNs caused by various types of electron tunnelling through the defects where $\tau_{\mathrm{c}}$ and $\tau_{\mathrm{e}}$ have the same polarity of bias dependence; and (b) in metallic conduction region near both electrodes. Defect's relative location within the electron tunnelling conduction region (TCR), $\mathrm{X}_{\mathrm{T}} / \mathrm{T}_{\text {ox }}$ TCR, can be extracted from eq.1 even though the absolute value of $\mathrm{T}_{\mathrm{ox}, \mathrm{TCR}}$ is unknown.

that the defects we examined are located in the electron tunneling region of the oxide, excluding the RTN caused by electrons tunneling through the defects. We also consider the metallic local conduction regions as part of the metal electrodes, thus $\mathrm{T}_{\mathrm{ox}}$ in eq. 1 is modified to $\mathrm{T}_{\mathrm{ox}, \mathrm{TCR}}$ and $\mathrm{X}_{\mathrm{T}} / \mathrm{T}_{\mathrm{ox}, \mathrm{TCR}}$ is the defect's relative spatial location within the electron tunneling conduction region (TCR). We will show in the following that clear correlations between RTN, defect and filament are revealed by using the technique we developed in this work after taking the above factors into account.

\subsection{Conductive Filament Profile}

The first key observation in this work, the spatial and 
energy profile of the CF, was achieved by statistically analyzing the defect spatial location and energy level during normal device operation cycles. We switched on and off the devices repeatedly between the LRS and HRS for a number of cycles, during each cycle the RTN measurement was carried out to extract the defects locations. A large number of defects can therefore be extracted during the cycling process. The measured defects profile at HRS during the cycling is shown in Fig. 3a, and a region with the least defects near, but not at, the BE can be clearly observed, with increasing defect number on either sides, which is named as the critical filament region, CFR, in this work.

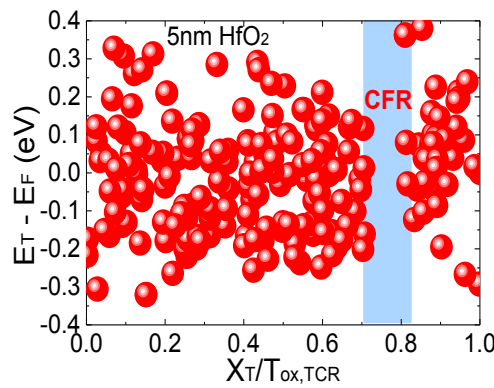

Fig.3 200 defects are detected at HRS during normal DC switching on/off cycles obtained in several devices which clearly shows the CFR near BE where the least defects are detected. Bias is incremented from $\pm 0.1 \mathrm{~V}$ to $\pm 0.35 \mathrm{~V}$, step $= \pm 0.01 \mathrm{~V}$, time $=3.5 \mathrm{~s} / \mathrm{step}$. $\mathrm{R}$ is measured at $0.1 \mathrm{~V}$. DC Vset $=1 \mathrm{~V}$, Vreset $=-1.6 \mathrm{~V}$, Icc $=150 \mathrm{uA}$. Note this defect profile does not provide information on actual defect density

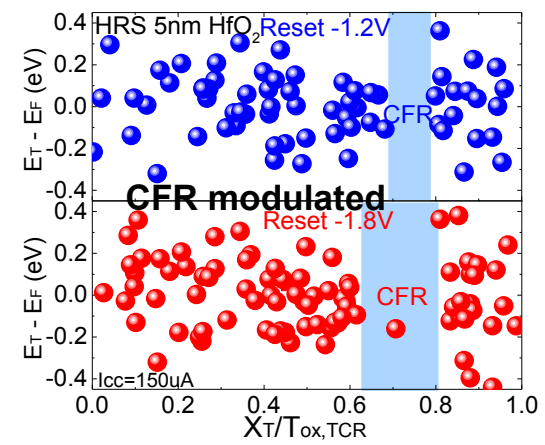

Fig.4 CFR is widened at higher Vreset, and R at HRS increases from $12.5 \mathrm{k} \Omega$ to $50 \mathrm{k} \Omega$. Vset= $1 \mathrm{~V}$.

Since the CFR has the least defects and hence the largest local resistance and electric field strength, the movement of defects into and out of the constriction should have the most significant impact on $\mathrm{CF}$ resistance, leading to $\mathrm{CF}$ modification and resistive switching. By applying the RTN technique, the modification of the CF at HRS under different operation conditions can be clearly observed, for the first time. As shown in Fig. 4, increasing Vreset from $-1.2 \mathrm{~V}$ to $-1.8 \mathrm{~V}$ widens the CFR mainly towards to the inner bulk of the oxide, thus increases the resistance at HRS from $12.5 \mathrm{k} \Omega$ to $50 \mathrm{k} \Omega$. As shown in Fig. 5, reducing the maximum compliance current during the set operation, Icc, from $150 \mathrm{uA}$ to $80 \mathrm{uA}$, widens the defect energy distribution due to the weaker regulation power caused by the lower set current. In order to observe the difference in CFR between HRS and LRS, a lower set voltage of $0.6 \mathrm{~V}$ is carefully chosen to result in a partial set operation where electron tunneling in the $\mathrm{CF}$ still dominates. This is because switching to a full-scale LRS at high Vset will lead to a full metallic conduction path where the RTN technique is no longer applicable. As shown in Fig.6, more defects in the CFR can be detected at the partial LRS, resulting in a weaker CFR and thus lower resistance. CFR can also be clearly observed in devices with the $\mathrm{HfO}_{2}$ thickness scaled further down to $3 \mathrm{~nm}$, confirming its capability of further scaling into the lower nanometer regime, as shown in Fig. 7.

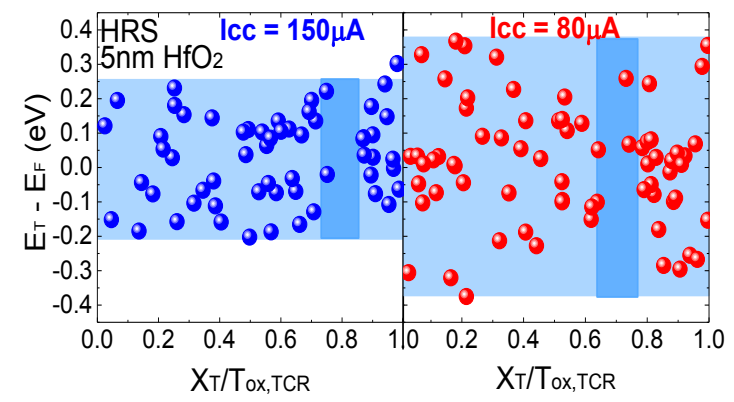

Fig.5 Defects have a wider energy distribution at a lower compliance $\mathrm{I}_{\mathrm{CC}}$, probably due to weaker regulation during SET. Vset $=1 \mathrm{~V}$, Vreset $=-1.6 \mathrm{~V}$

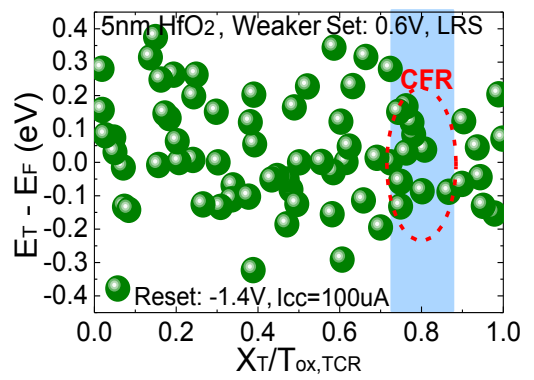

Fig.6 At a partial LRS where e-tunnelling dominates, more defects can be detected within CFR.

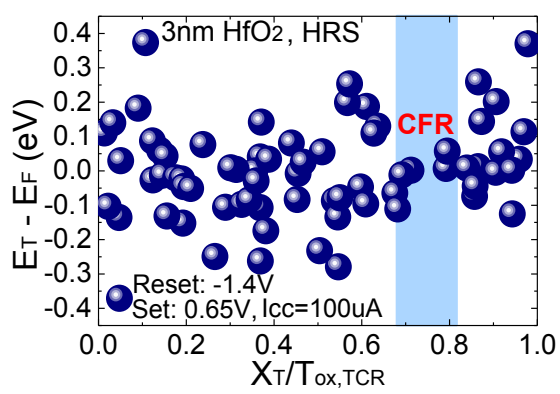

Fig.7 CFR is also observed in devices with $3 \mathrm{~nm} \mathrm{HfO}_{2}$ layer near the BE. 


\subsection{RTN based Defect movement Tracking (RDT)}

We further developed the RTN technique to enable the tracking of individual defect movements, so that the missing information on defect movement can be experimentally obtained and directly correlated with $\mathrm{CF}$ modification and resistive switching. Fig.8a illustrates the typical RDT test procedure. During the bias sweeping of RTN measurement at HRS, irregular current jumps, either upwards or downwards, can be observed, which is associated with the simultaneous change in device resistance, supporting that the current jumps are caused by modification of the CF. The defect location extracted from the RTN before and after the current jumps also change simultaneously, indicating that the $\mathrm{CF}$ modification is induced by the defect movement.
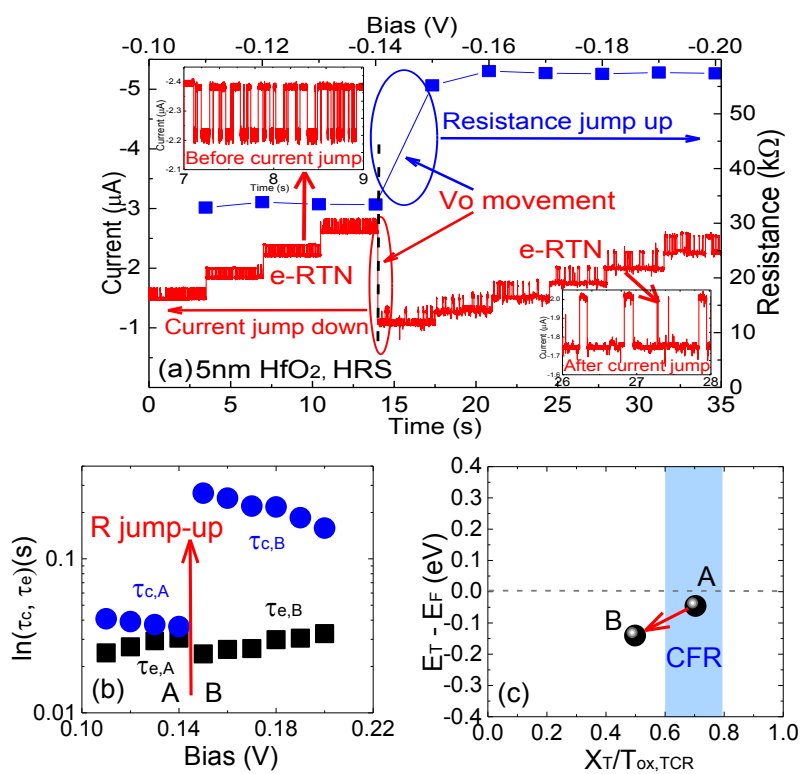

Fig.8 RTN based defect tracking (a) RTN signals consist of both Vo movement and e-RTN. Bias is incremented from $\pm 0.1 \mathrm{~V}$ to $\pm 0.35 \mathrm{~V}$ $(-0.1 \mathrm{~V} \sim-0.2 \mathrm{~V}$ is shown), step $= \pm 0.01 \mathrm{~V}$, time $=3.5 \mathrm{~s} / \mathrm{step} . \mathrm{R}$, measured at $0.1 \mathrm{~V}$ at each step, changes simultaneously with Vo movement. DC Vset $=1 \mathrm{~V}$, Vreset $=-1.6 \mathrm{~V}$, Icc $=150 \mathrm{uA}$. (b) $\tau_{\mathrm{c}}$ and $\tau_{\mathrm{e}}$ measured before/after the $\mathrm{R}$ jump, from which (c) defect movement in $\left(\mathrm{X}_{\mathrm{T}}, \mathrm{E}_{\mathrm{T}}\right)$ is extracted.

To further demonstrate the correlation between defect movement and filament modification, amplitude of $\Delta$ Iread/Iread disturbs are analyzed against $X_{T}$ and $E_{T}$ before and after Vo movements. Fig.9a shows that the largest current increases (jump-up) are associated with Vo moving towards CFR when CFR is the destination of defect movements. In contrast, the largest current decreases (jump-down) are caused by Vo moving out of CFR, as shown in Fig.9b. These results highlight the importance of the CFR in determine the resistance of the
RRAM devices, when the location of defect movements is farther away from the CFR, its impact on device resistance is insignificant.
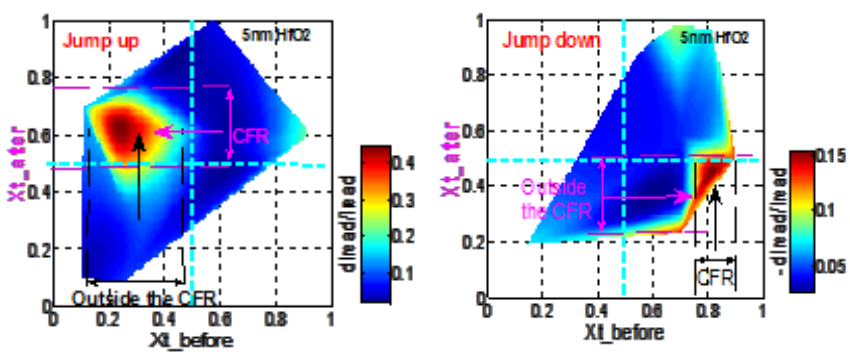

Fig.9 Iread/Iread vs XT before and after Vo movement. (a) The largest Iread increase occurs when Vo moving towards CFR (2nd quadrant). (b) the largest Iread decrease happens when Vo moves out of CFR (4th quadrant). No jumps are observed in the blank (white) region. HRS.

\section{Conclusions}

For the first time, An RTN based technique has been developed that can monitor the defect movement and filament alteration in RRAM devices. Critical filament region has been identified during switching operation at various conditions, and more importantly, the CFR in nanoscale devices plays the critical role in determining the resistive switching. This technique provides a useful tool for RRAM technology development.

\section{Acknowledgments}

This work was supported by the Engineering and Physical Science Research Council of UK under the grant no. EP/M006727/1. The authors would like to thank colleagues at IMEC, Belgium, for supply of test samples used in this work and fruitful discussions.

\section{References}

[1] Wong et al, IEEE proc., 2012.

[2] Govoreanu et al, IEDM, 2011.

[3] R. Degraeve et al, VLSI, 2012.

[4] Cong et al, IEEE Trans. VLSI Syst. 2014, 22 (4), 864-877.

[5] Burr et al, IEEE Trans. Elect. Dev, 2015, 62, 3498-3507.

[6] Waser et al, Adv. Mater., 2009, 21, 2632-2663.

[7] Celano, et al, IEDM, 2013.

[8] Kwon, et al, IRPS, 2014.

[9] Moors et al, ACS Nano, 2016, 10, 1481-1492.

[10] Raghavan et al, VLSI 2013.

[11] Kirton et al, Advances in Physics, 1989.

[12] Chang et al, IEDM 2008. 